Apidologie, 1984, 15 (2), 189-204

\title{
FUNKTIONSMORPHOLOGISCHE BEFUNDE BEI DER KOPULATION DER HONIGBIENE (APIS MELLIFERA L.)
}

\author{
Gudrun KOENIGER \\ Institut für Bienenkunde (Polytechnische Gesellschaft) \\ Karl-von-Frisch-Weg 2, 637 Oberursel
}

\section{ZUSAMMENFASSUNG}

Königinnen mit künstlich geöffneter Stachelkammer wurden an einem Mast befestigt und freifliegenden Drohnen zur Paarung geboten. Bei diesem Versuch stoppt die Kopulation häufig in einem Stadium, in dem der Drohn seinen Endophallus bis zu den Skleriten der Cervix evertiert hat. Die verhängten Paare wurden eingefroren und untersucht.

In diesem Kopulationsstadium sind die Sklerite der Cervix in die Vagina cingeführt. Die Cornua sind in der Regel nur partiell ausgestülpt und verlaufen entlang der behaarten Chitinfalte zwischen Bursa copulatrix und Stachelkammer zur Dorsalseite der Königin. Das clorsale triangulare Haarfeld liegt etwa in Höhe dieser Chitinfalte, das ventrale haarige Rautenfeld schräg unterhalb des Stachelapparates.

Der Stachelapparat ist dorsal und cranial verschoben. Seine drei Chitinplatten haben sich um ihre dorso-ventrale Achse gedreht. Dabei werden die Falten der chitinösen, häutigen Auskleidung der Bursa copulatrix so verschoben, daß die Vagina trichterförmig geöffnet wird.

Der Ablauf der Kopulation wird in Hinblick auf diese Befunde diskutiert.

\section{EINLEITUNG}

«Der Hochzeitsflug und die Begattung der Bienenkönigin gehören... zu jenen Problemen, mit welchen sich die Bienenforscher und führenden Imker des letzten Jahrhunderts auf das eifrigste beschäftigt haben $\gg(\mathrm{FYG}, 1947$, S.76). Die Literatur ist so umfangreich, daß sich z.B. Arnhart (1938) außerstande sieht, alle über die Begattung und das Begattungszeichen erschienenen Schriften anzuführen. Allein aus der «Nördlinger Bienenzeitung» liegen ihm 70 Berichte vor, gesammelt hat er über 175 Veröffentlichungen zu diesem Thema.

Trotz des großen Interesses entziehen sich Drohnen und Königinnen während der natürlichen Paarung weiterhin jeglicher genauer Beobachtung, da diese nur in 
freiem Flug hoch in der Luft stattfindet. Dennoch konnten in den Jahren von etwa 1940 bis heute durch viele gezielte Untersuchungen auf indirektem Wege die Kenntnisse über die Paarungsbiologie wesentlich erweitert werden. So wissen wir heute, daß sich Königinnen im allgemeinen auf Drohnensammelplätzen, vorzugsweise in einer Entfernung von 2-3 km von ihrem Stock, paaren (Ruttner F.u.H., 1972). Sie fliegen im Alter von 6-10 Tagen aus und werden während des Fluges von mehreren Drohnen begattet (RoBerTs, 1944 ; RUTTNER, 1954 ; TABER, 1954 ; Triasko, 1956; WoYke, 1955).

Die Drohnen werden sowohl optisch angelockt, als auch olfaktorisch durch die Sekrete der Mandibeldrüsen ( 9 OD) und der Tergittaschendrüsen (GARY, 1962 ; Pain und Ruttiner, 1963 ; Renner und Vierling, 1977). Die Königinnen unternehmen zwischen 1 und 3 Hochzeitsflüge.

Durch neue technische Möglichkeiten gelang es kürzlich, das Kopulationsverhalten von freifliegenden Drohnen mit fixierten Königinnen zu filmen (Koeniger et al., 1979 ; Koeniger, 1981).

Die Drohnen reiten dorsal auf die Königin auf, umklammern ihr Abdomen mit den Beinen und führen ihren Endophallus in die Königin ein. Bei dieser Versuchsanordnung stoppt die Eversion des Endophallus im allgemeinen in einem Stadium, in dem gerade der Anfang der Cervix ausgestülpt ist. Das Paar bleibt miteinander verhängt. Dadurch bot sich eine Möglichkeit, die Lage des Endophallus in der Königin zu untersuchen.

Bisher wurden die Hypothesen über die Lage des kompliziert gebauten Endophallus und über die Bedeutung seiner Anhänge auf Grund von Vergleichen der anatomischen Verhältnisse des Endophallus mit denen der Bursa copulatrix entwickelt. Sie kommen zu unterschiedlichen Ergebnissen. Besonders kontrovers wird die Lage der Cornua diskutiert. Sehr häufig wird die Meinung vertreten, daß sie in die Seitentaschen der Bursa copulatrix eindringen (BisHop, 1920 ; SNOdgrass, 1956, u.a.). Nach anderen Autoren hängen sie jedoch frei an den Seiten der Kloake (ARNHART, 1938) oder sie sollen gar nicht in die Königin eingeführt werden, sondern ihr Abdomen analog zu den Parameren anderer Insekten von außen umschließen (Woyke, 1955).

Durch eine Untersuchung von verhängten Paaren scheint die Klärung dieser Fragen möglich.

\section{MATERIAL UND METHODE}

Die Stachelkammer der Königin wurde durch die Fixierung der Gelenke zwischen dem letzten Tergit und Sternit mit Klebstoff offengehalten. Danach wurde die Königin längs an einen dünnen Draht geklebt. Dadurch kann ihr Abdomen in die Flughaltung gebogen werden. Bei schönem 
Wetter wurde sie am Nachmittag den Drohnen auf einem 6-10 m hohen Mast am Drohnensammelplatz zur Paarung geboten (Koeniger G., 1979). Bei der Befestigung am Mast muß darauf geachtet werden, daß sich die Königin in einer waagerechten Stellung befindet.

Nach der Verhängung wurde der Mast verkürzt und das Paar sofort auf $\mathrm{CO}_{\text {.-Schnee }}$ $\left(-80^{\circ} \mathrm{C}\right)$ eingefroren.

In gefrorenem Zustand wurden die Paare in Agar eingebettet, um eine Verschiebung des Drohns während der Präparation möglichst gering zu halten.

Zunächst wurde die Königin von der Ventralseite her aufpräpariert, um die Lage des Endophallus in der Vagina und den lateralen Taschen der Bursa copulatrix zu verfolgen. Danach wurde die Präparation von der Seite fortgesetzt. Zum Schluß wurde der Drohn ganz entfernt und die Stellung des Stachelapparates analysiert. Insgesamt konnten auf diese Weise 14 Paare untersucht werden.

\section{ERGEBNISSE}

Alle untersuchten Paare sind äußerlich im gleichen Kopulationsstadium fixiert. Der Drohn ist paralysiert und nach hinten weggekippt. Dadurch zeigt seine Ventralseite nach oben und liegt fast in einer Ebene mit der Dorsalseite der Königin (Abb. 1).

Bei 7 Königinnen lag der Endophallus schräg in der Königin. Durch die Fixierung am Draht können die Königinnen keine Drehung um ihre Längsachse ausführen, während die Drohnen auf äußere Reize frei reagieren. Bei der natürlichen Paarung verhalten sich Drohn und Königin wahrscheinlich gleichsinnig. Deshalb wird im folgenden nur die Lage des Endophallus beschrieben, bei der sich Drohn und Königin in derselben dorso-ventralen Ebene befinden.

\section{A. Lage des Endophallus}

Der Endophallus ist auf seiner Dorsalseite bis zum triangularen Haarfeld (TF) evertiert, während an der Ventralseite die V-förmigen Sklerite der Cervix (SC) zu sehen sind (Abb. 2d und 4a). Das triangulare Haarfeld befindet sich in Höhe der mit dornenartigen Haaren besetzten Chitinfalte (HF) and der Ventralseite der Königin, die zwischen der Bursa copulatrix und der äußeren Stachelkammer verläuft. Der ventrale Teil der Cervix mit den Skleriten ist in die Vagina eingeführt. Es konnte nicht mit Sicherheit festgestellt werden, ob die Cervix cranial oder caudal von der Valvula vaginalis endet. Die Cornua (CO) sind bis auf eine Ausnahme nur zum Teil ausgestülpt. In keinem Fall dringen sie in die lateralen Taschen der Bursa copulatrix ein, sondern biegen entlang der behaarten Chitinfalte der Königin in Richtung des Stachelapparates. In dem Fall, in dem die Cornua vollständig 
ausgestülpt sind, treffen sich die Spitzen ventral vom Stachel. Beim Herauslösen des Endophallus bleibt ein Teil der orangefarbigen Substanz, die die Cornua überzieht, in den Dornen der Chitinfalte hängen.

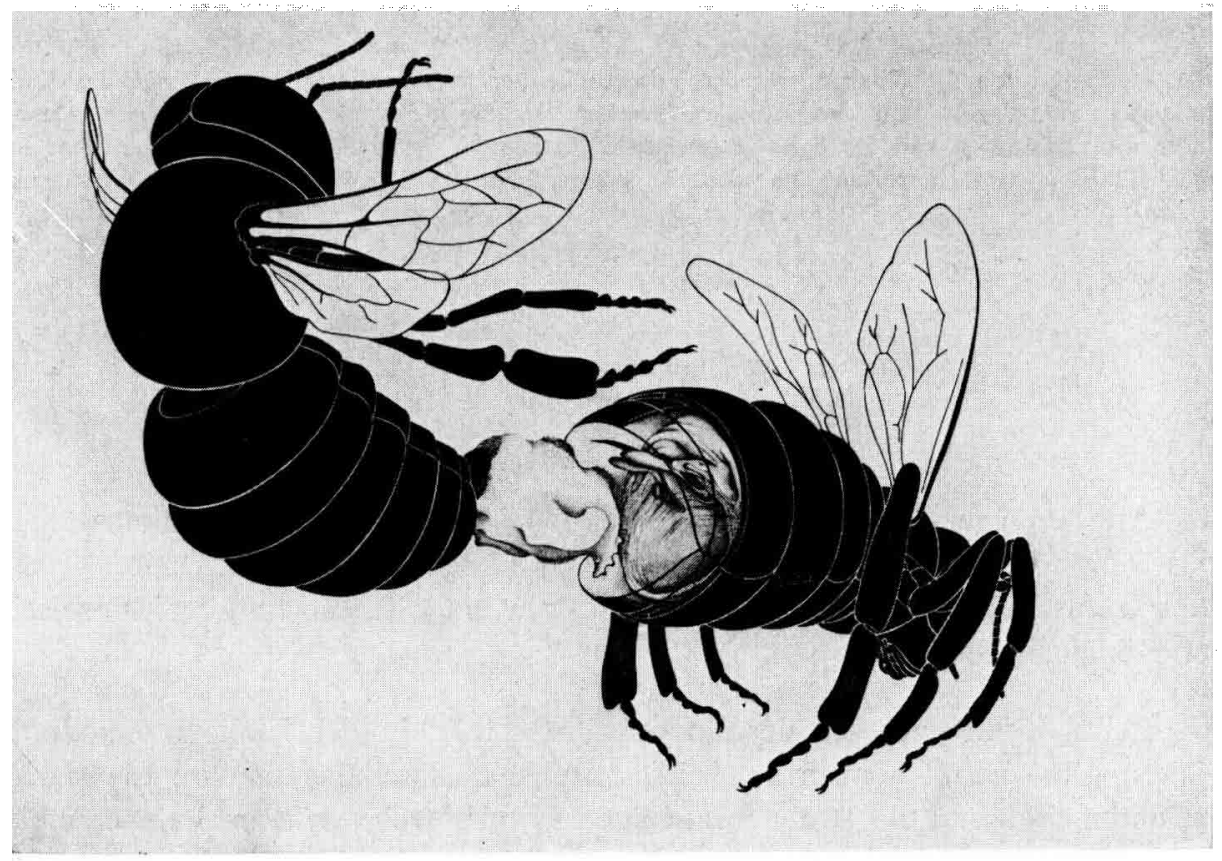

ABв. 1. - Experimentelle Trennung eines verhängten Paares (Zeichnung von M. KREUder)

FIG. 1. - Experimenial detachment of a copulating pair (Drawing from M. KREUDER)

Das ventrale haarige Rautenfeld (RF) des Endophallus liegt schräg unterhalb des Stachelapparates und scheint diesen dabei nach cranial und dorsal zu drücken. Der Stachel verläuft dicht über dem Endophallus. Seine Krümmung ist so beschaffen, daß die Spitze zwar dicht aufliegt, aber nicht in den Endophallus einsticht.

Durch die dünne Haut des Endophallus ist zu erkennen, daß die Chitinspangen des Begattungszeichens zwischen dem Ansatz der Cornua liegen, und daß sich der Bulbus bereits mit Mucus gefüllt hat (Abb. 4a). 


\section{B. Änderung der Stellung des Stachelapparates während der Paarung}

\section{Ruhelage des Stachelapparates}

Nach der Entfernung eines Teils des letzten Tergits und Sternits sieht man, daß die 3 Platten des Stachelapparates, die oblonge (OP), die quadratische (QP) und die Stigmenplatte (SP) längs (cranio-caudal) in der Königin liegen (Abb. 2a,b). Die Rami des Stachels liegen über der Chitinfalte, die zwischen der Stachelkammer und der Bursa copulatrix verläuft. Die Stachelspitze (ST) ruht in der Kerbe des letzten Sternits, die Stachelscheiden (STS) befinden sich dorsal vom Stachel.

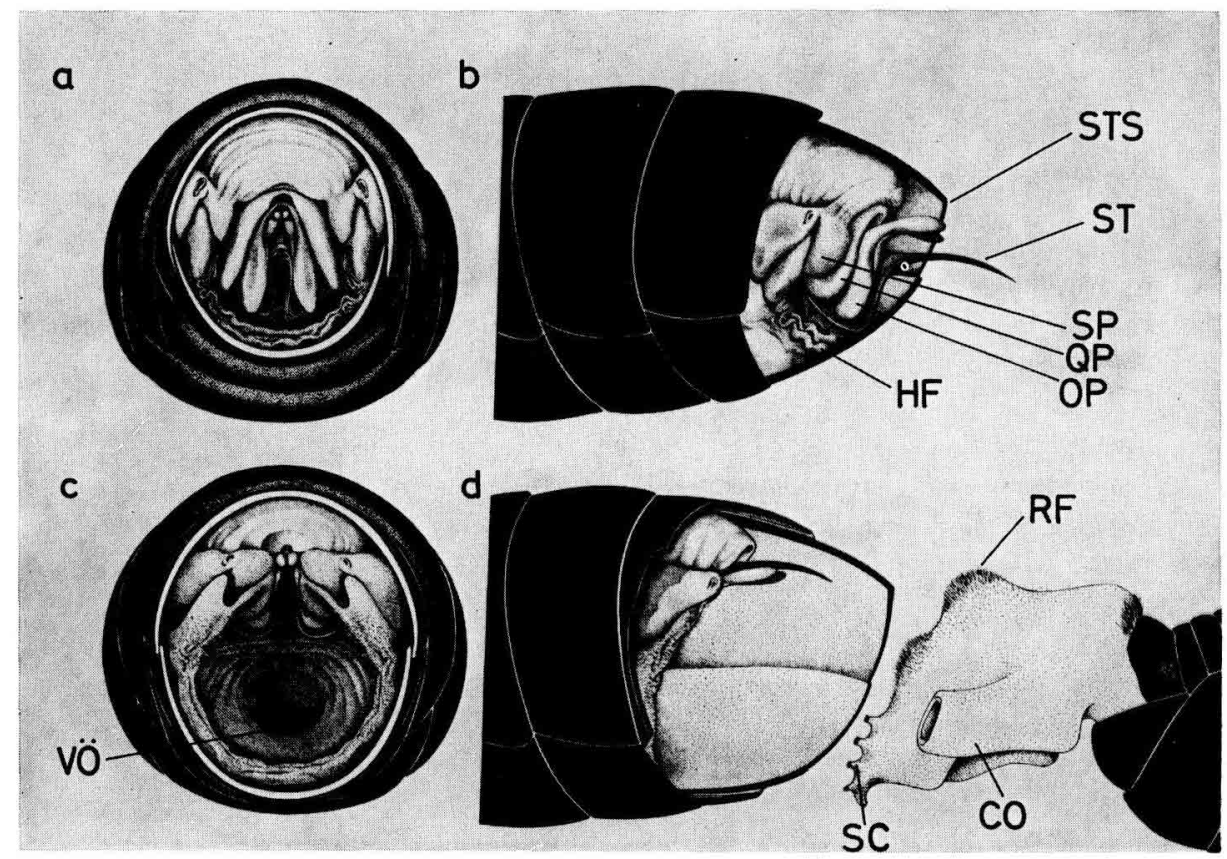

Aвв. 2. - Lage des Stachelapparates der Königin vor und während der Paarung (Zeichnung von M. KREUder)

a) Querschnitt vor der Paarung.

b) Längsschnitt vor der Paarung (Stachel exponiert).

c) Querschnitt während der Paarung.

d) Längschnitt wäh:end der Paarung.

Erklärungen für die Abkürzungen siehe Seite 194.

FIG. 2. - Position of the sting apparatus of a queen before and during copulation (D:awing from M. KREUDER)

a) Cross section before mating.

b) Longitudinal section before mating (sting exposed).

c) Cross section during mating.

d) Longitudinal section during mating.

Key to abbreviations see page 194 . 


\section{KEY TO ABBREVIATIONS USED IN FIGURES}

English

BE Bulb of endophalus; penis bulb.

B1 The part of the bulb which is strengthened by the chitinized plates and the bow of the bulb.

B2 Thin-walled part of the bulb.

BC Bursa copulatrix.

BP Bursal pouch.

BZ Mating sign.

CD Dorsal chitinized plates.

CE Cervix.

CL Lateral chitinized plates of the bulb.

CO Cornua.

CS Connective substance; «semi-transparent thickening of the cuticular intima» (Snodgrass 1956, p. 296), ,

E Edge of connective substance partly concealing the posterior opening of the bulb of the endophallus.

EDP Posterior end of the ejaculatory duct.

FL Fimbriate lobe.

HF Hairy rim.

LO Lateral oviduct.

LP Lamina parameralis; upper clasper.

M Covering of the mating sign.

MO Median oviduct.

MS Mating sign.

MU Mucus.

OB Posterior opening of the bulb.

OC External opening of the cervix.

OP Oblong plate.

PV Penis valve.

QP Quadrat plate.

RF Ventral hairy field.

SB Sac of the bulb.

SC Sclerites of cervix.

SE Semen.

SP Spiracular plate.

ST Sting.

STS Sting sheath.

TF Triangular hairy field.

VE Vestibulum (base of endophallus).

Vö Opening of vagina.

WD Doming of the bulb wall, surrounding the pointed ends of the dorsal plates.

\section{German}

Bulbus.

Teil des Bulbus verstärkt durch die Chitinplatten und die Bulbusschleife.

Dünnwandiger Teil des Bulbus.

Bursa copulatrix.

Seitentasche der Bursa copulatrix.

Begattungszeichen.

Dorsale Chitinplatten.

Cervix.

Chitinplatten des Bulbus.

Cornua.

Verbindungsmasse.

Rand der Verbindungsmasse, die hintere Bulbusöffnung teilweise verdeckend.

Hinteres Ende des Ductus ejaculatorius.

Gefiederter Anhang.

Haarige Chitinfalte.

Lateraler Ovidukt.

Deckschuppe.

Hülle des Begattungszeichens.

Medianer Ovidukt.

Begattungszeichen.

Mucus.

Hintere Öffnung des Bulbus.

Aussere Öffnung der Cervix.

Oblonge Platte.

Deckplatte.

Quadratische Platte.

Rautenfeld.

Bulbussack.

Sklerite der Cervix.

Sperma.

Stigmenplatte.

Stachel.

Stachelscheiden.

Triangulares Haarfeld.

Vestibulum (Basis des Endophallus).

Öffnung der Vagina.

Vorwölbung der Bulbuswand, die Enden der dorsalen Chitinplatten umfassend. 


\section{Lage des Stachelapparates während der Paarung}

Ein ganz anderes Bild zeigt der Stachelapparat während der Paarung. Nach Entfernung der letzten äußeren Sklerite bis zum Rand des vorletzten Segmentes ist der Stachelapparat von der Seite kaum noch zu erkennen, er ist bis unter die Sklerite des 5. Hinterleibsegments verlagert (Abb. 2d). Gleichzeitig wurde er geringfügig gedreht, so daß der Stachel, den After verdeckend, dicht am Tergit entlang läuft. Die Stachelscheiden liegen in gleicher Höhe oder sogar ventral vom Stachel.

Die 3 Platten des Stachelapparates sind um ihre dorso-ventrale Achse gedreht. Dabei klappen die oblonge und die quadratische Platte zum Teil über die Basis des Stachels (Abb. 1 u. 2c). Die Stigmenplatte wird in einer verdickten Chitinleiste caudal des Stigmas geknickt, so daß das ursprünglich caudal gelegene Chitinteil quer steht, während der größere, mit dem Stigma versehene Teil die Drehung nur in einem geringeren Maße vollzieht.

Durch diese Verlagerung der Chitinplatten wird die innere, häutige Auskleidung der Bursa copulatrix stark beeinflußt. Die in Ruhestellung in Falten gelegte behaarte Chitinfalte wird vor allem durch die Drehung der Stigmenplatte seitlich gespannt, durch das gleichzeitige öffnen des letzten Sternits auch in dorsoventraler Richtung. Dadurch werden die Hautpartien um die Vaginalöffnung auseinandergezogen, so daß die Vagina trichterförmig offen daliegt (Abb. 2c).

\section{DISKUSSION}

Durch die Fixierung des Abdomens einer Königin an einem Draht und die Öffnung der Stachelkammer gelingt es, die Flughaltung der freifliegenden Königin zu imitieren. Man kann davon ausgehen, daß die Paarungen mit diesen Königinnen denen der freifliegenden sehr ähnlich sind.

Die Drohnen kopulieren mit Attrappen, sofern diese eine Öffnung von 2-4 mm im Durchmesser aufzeigen (GARY und MARSTON, 1971). Sie verhängen sich auch mit Königinnen, die waagerecht mit der Ventralseite nach oben am Mast befestigt sind, wenn die Stachelkammern offen gehalten werden. Deshalb hängt die Lage des Endophallus davon ab, wie die Königin vor der Paarung befestigt wird. In dieser Arbeit wurde davon ausgegangen, daß sich Drohn und Königin bei der natürlichen Paarung in derselben dorso-ventralen Ebene befinden. Nur diese Stellungen wurden zur Auswertung herangezogen.

Unter Berücksichtigung der Ergebnisse aus dem Paarungsfilm (KoENIGER, 1981), der Lage des Endophallus in der Königin während der ersten Phase der Verhängung und der künstlichen Eversion des Endophallus (WoyKE und RuTTNER, 1958) soll versucht werden, den Ablauf der Kopulation zu rekonstruieren. 


\section{Greifen der Königin}

Der Drohn berührt das Abdomen der Königin zunächst mit den beiden vorderen Beinpaaren an ihrer Dorsalseite. Dann ergreift er sie auch mit dem hinteren Beinpaar an der Überlappung der 4.-5. Tergite über die Sternite, so daß die Königin in einer dorso-ventralen Klammer gehalten wird (KoENIGER et al., 1979). Das hintere Beinpaar ist mit besonderen Hafthaaren ausgestattet (RUTTNER, 1975). Beim Einbiegen seines Abdomens bleibt ein Zwischenraum zur Königin, so daß er sie nur mit dem genitalen Sinnesfeld (RutTNer, 1962) berührt. Damit ertastet er wahrscheinlich die Beschaffenheit der Hinterleibsspitze bzw. der Stachelkammer der Königin.

\section{Eversion des Endophallus bis zur Cervix}

Die Eversion des Endophallus beginnt mit dem Austreten des haarigen Rautenfeldes. Hat die Königin ein Begattungszeichen (Abb. 3), berührt er dieses mit dem Rautenfeld. Bei der weiteren Eversion wird das Begattungszeichen so in der Königin gedreht, daß die ursprünglich dorsal in der Königin gelegene Seite nun am haarigen Rautenfeld des Vestibulums hängen bleibt. Wahrscheinlich hat der orangene Belag, der das Begattungszeichen überzieht, eine Klebefunktion.

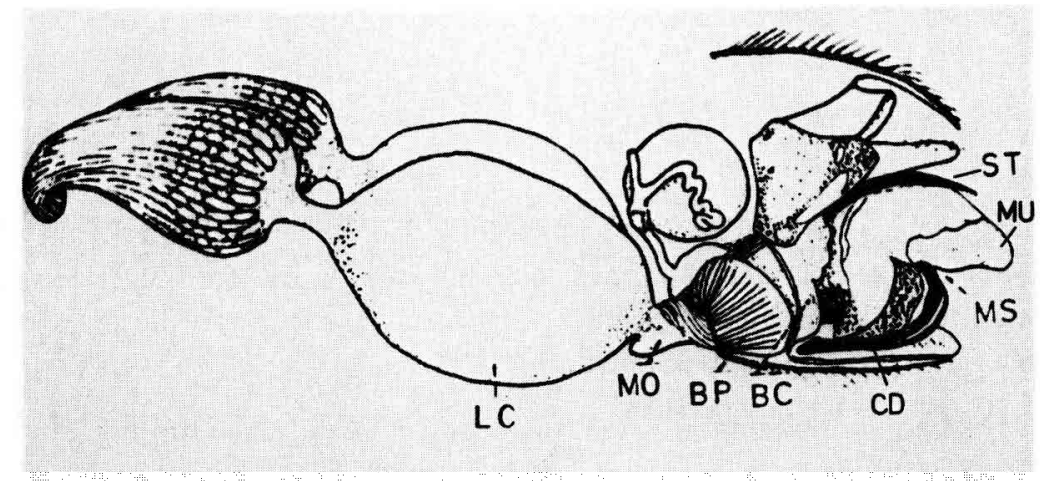

Aвв. 3. - Lage des Begattungszeichens in der Königin (nach WoyKe und RUTTNER, 1958) Erklärungen für die Abkürzungen siehe Seite 194.

Fig. 3. - Position of the mating sign in the queen (after WoYKe and RUTTNER, 1958) Key to abbreviations see page 194.

Bei der Drehung des Begattungszeichens müssen dessen Chitinspangen am Stachelapparat entlang gleiten. Dabei wird der Stachelapparat wahrscheinlich angehoben und die Gefahr, daß der Endophallus durch den Stachel verletzt wird, wird verringert. Mit der vollständigen Ausstülpung des Vestibulums ist die Drehung des Begattungszeichens beendet. 
Für die Eversion des Endophallus pumpt der Drohn durch Kontraktion der Abdominalmuskeln soviel Hämolymphe und Luft hinein, daß er paralysiert wird, was an dem plötzlichen Stillstand der Flügel zu erkennen ist. Dabei schwingt der Drohn von der Dorsalseite nach hinten. In diesem Stadium befindet sich nur ein kleiner basaler Teil des Vestibulums außerhalb der Königin. Die Stachelkammer und die Bursa copulatrix sind stark gedehnt, weil der Umfang des Endophallus in Höhe der dorsalen Höcker des Vestibulums bis zum ventralen Rautenfeld sehr groß ist. In dieser Situation ist der Endophallus sehr fest, da sich der Bulbus (BE) mit Chitinspangen (CD), Bulbusschleife und Mucus (MU) innerhalb des Vestibulums befindet (Abb. 4a). Zusätzlich liegt meist noch das Begattungszeichen des Vorgängers zwischen dem Rautenfeld und dem Stachelapparat.
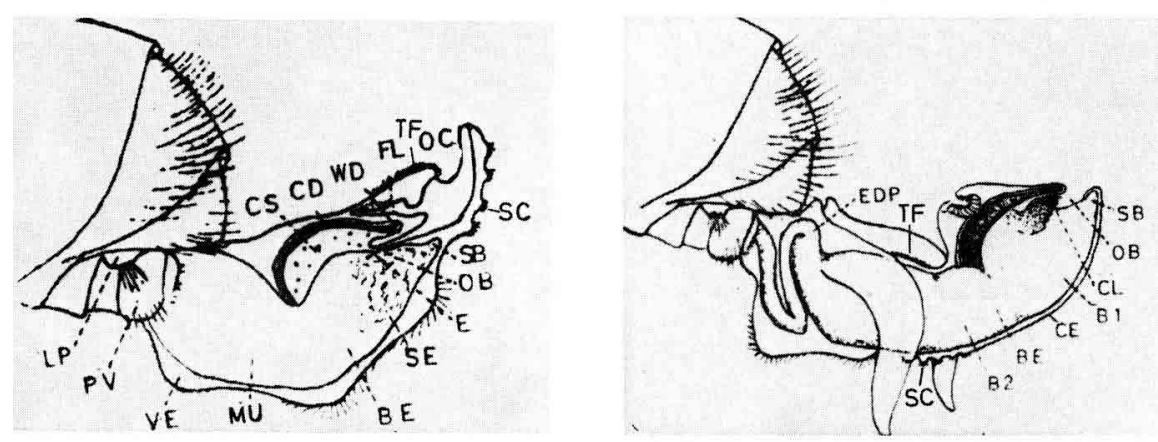

Aвв. 4. - Eversionsstadien des Endophallus (nach WoYke und RutTner, 1958) Erklärungen für die Abkürzungen siehe Seite 194.

FIG. 4. - Stages in the eversion of the endophallus (after WoyKe and RUTTNER, 1958) Key to abbreviations see page 194.

Die Cornua sind nur zum Teil ausgestülpt. Sie liegen seitlich an der behaarten Chitinfalte der Königin und erhöhen zum einen die seitliche Ausdehnung, zum anderen unterstïtzen sie die Festigkeit der Verhängung, da sie mit ihrem orangenen Belag in den Haaren haften bleiben. Der orangene Belag scheint aus der gleichen Substanz zu bestehen, wie der des Begattungszeichens (Woyke und RutTNer, 1958).

Die Cervix dringt mit ihren Querleisten in die geöffnete Vagina ein. Die Verhängung ist in diesem Stadium so fest, daß man Drohn und Königin kaum voneinander trennen kann, ohne einen von ihnen zu verletzen.

\section{Vollständige Eversion des Endophallus}

Bei dem weiteren Ablauf der Paarung muß sich die Königin aktiv beteiligen, da der Drohn bereits paralysiert ist. Der Bulbus mit dem Begattungszeichen 
$m u ß$ in die enge Cervix eintreten, die dadurch stark gedehnt wird. Im Film über die Paarung dauert es einige Zeit, bis diese Eversion der Cervix einsetzt. Durch die Verklebung der Stachelkammer ist die Königin wohl in ihrer Bewegungsmöglichkeit eingeschränkt. Wahrscheinlich übt die Königin unter natürlichen Bedingungen mit der Muskulatur des Stachelapparates und der äußeren Sklerite einen starken Druck auf den Endophallus aus. Wic sich die Lage des Endophallus in der Königin dabei verändert, konnte nicht untersucht werden, so daß es unklar bleibt, ob die Cervix über ihre Sklerite hinaus in die Vagina eindringen kann. Die Spermaabgabe erfolgt unter künstlichen Bedingungen kurz vor der Eversion des Bulbus (Abb. 4b).

Im Paarungsfilm ist zu sehen, wie das Vestibulum aus der Königin heraustritt, an seinem Rautenfeld klebt das Begattungszeichen seines Vorgängers (Abb. 5). Die Hörnchen treten voll ausgestülpt ohne ihren orangenen Bezug aus. Die orangene Substanz bleibt an der behaarten Chitinfalte in der Königin kleben.

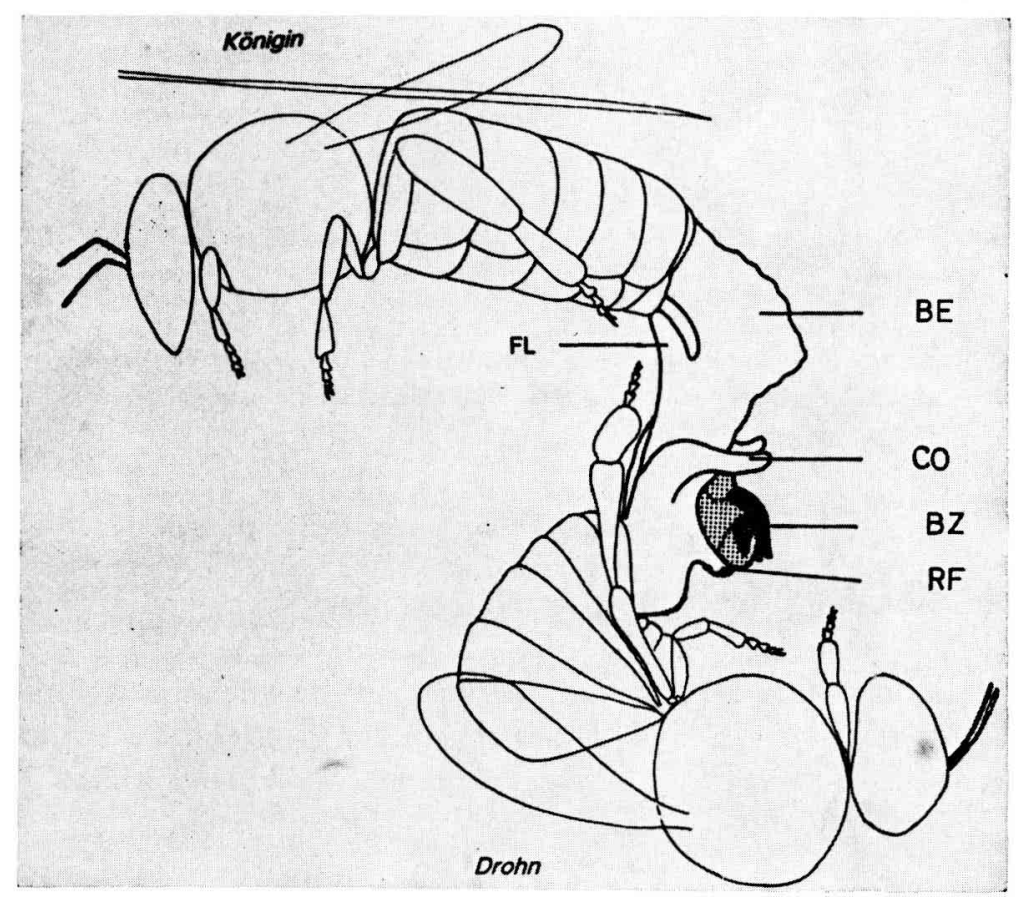

Авв. 5. - Letztes Kopulationsstadium

Erklärungen für die Abkürzungen siehe Seite

Fig. 5. - Last stage of copulation

Key to abbreviations see page

In dieser Phase stoppt die Eversion wiederum für einige Zeit, der distale Teil des Federanhangs (FL) und der Bulbus stecken noch in der Königin. Es ist also 
auch in diesem Stadium eine Aktivität der Königin notwendig. Nach einiger Zeit löst sich dann der Drohn unter Zurücklassung seines Begattungszeichens von der Königin. Dabei wird auch der Bulbus völlig evertiert, wie die Funde von Drohnen unter einem Drohnensammelplatz beweisen (Triasko, 1957 ; BötTCHER, mündliche Mitteilung).

Bei der Diskussion des kompliziert gebauten Endophallus sollte nicht nur seine Funktion für die feste Verhängung während der Paarung im freien Flug berüicksichtigt werden. Ein anderer Aspekt liegt darin, daß der Endophallus die Entfernung zwischen der Öffnung der Stachelkammer am Hinterende der Königin und der Vaginalöffnung überbrücken muß, die nach ARNHART (1938) $1,5 \mathrm{~mm}$ beträgt. Dabei muß das Verhalten der Königin in einer spezifischen Art erfolgen, denn in der Normallage ist die Bursa copulatrix durch den Stachelapparat verdeckt und die Stachelspitze ragt genau bis zur Hinterleibsöffnung.

\section{Lage des Stachelapparates}

Die Lage des Stachels während der Paarung spielt eine wichtige Rolle. Bei der Kopulation verschiebt und dreht sich der Stachelapparat so, daß Stilett und Lanzetten zwischen After und Tergit einerseits und dem Endophallus andererseits verläuft. Dabei entspricht die Krümmung des Stachels genau denen des Endophallus und des Tergits. Wäre der Stachel so gerade geformt wie bei der Arbeiterin, könnte sich die Königin bei dieser Stellung leicht selbst verletzen, eine stärkere Krümmung hätte möglicherweise einen Stich in den Endophallus zur Folge. Diese Frage hatte schon von Buttel-ReePEN (1919) diskutiert. Nach Zander (1922) « eignet sich die gekrümmte Gestalt vorzüglich zum Festhalten des Begattungszeichens ».

Trotz der veränderten Lage des Stachels kann es zu Verletzungen der Partner während der Paarung kommen. Von Königinnenzüchtern wird berichtet, daß sich die Königinnen manchmal mit ihrem Stachel in der Afterpapille oder im Tergit verhaken (GöTz, mündliche Mitteilung), sodaß die Königin ihre Eier nicht normal ablegen kann. Eine andere Legestörung wird beschrieben, bei der der Stachel in das Begattungszeichen eingedrungen ist, wodurch dieses nicht mehr aus der Königin entfernt werden kann (ARNART, 1935). Während der Kopulation kann sich die Stachelspitze auch in den Endophallus bohren. Die Folge ist ein Turgorverlust des Endophallus, der die vollständige Eversion und damit die Trennung von Drohn und Königin verhindert (WOYKE und RuTTNER, 1958). So läßt sich die Krümmung des Königinnenstachels unter anderem auch als eine Anpassung an die Paarung deuten.

Die Drehung des Stachelapparates bewirkt eine Änderung bei der chitinösen, häutigen Auskleidung der Stachelkammer und der Bursa copulatrix. Die Haut wird in einer Weise gespannt, daß die Hautfalten, die in Ruhelage die Vaginal- 
öffnung verdecken, auseinander gezogen werden. Auf diese Weise entsteht eine trichterförmige Öffnung der Vagina, die das Eindringen des Endophallus ermöglicht. Wahrscheinlich ist die Bewegung des Stachelapparates nicht der einzige Mechanismus, der an der Öffnung der Vagina beteiligt ist. Die starke Muskulatur, die an den Seitentaschen der Bursa copulatrix ansetzt, könnte ebenfalls in funktionellem Zusammenhang mit der Öffnung der Vagina stehen. In diesem Fall können die lateralen Taschen der Bursa copulatrix als Chitinausstülpung zum Ansatz dieser Muskelstränge angesehen werden.

Der Fragenkomplex, wie die Muskeln des Stachelapparates und der Genitalregion zusammenarbeiten, muß in künftigen anatomischen Untersuchungen geprüft werden.

Eingegangen im April 1984.

\author{
RÉSUME \\ ETUDE MORPHO-FONCTIONNELLE DE LA COPULATION \\ CHEZ L'ABEILLE (APIS MELLIFICA L.)
}

Des mâles volant librement ont pu s'accoupler avec des reines dont la chambre de l'aiguillon était maintenue ouverte par le blocage de l'articulation entre le dernier tergite et le sternite (Koeniger et al., 1979). Au cours de cette expérience le processus d'accouplement s'est le plus souvent arrêté après l'éversion de l'endophallus jusqu'aux sclérites du col (SC, Fig. 2d et 4a). Dans cette position le couple est si fortement attaché qu'il a pu être congelé en copulation $\left(-80^{\circ} \mathrm{C}\right)$. Pour cela le couple a été enrobé dans une gelée d'agar afin d'éviter le déplacement de l'endophallus.

\title{
Résultats
}

\section{Position de l'endophallus}

Les sclérites du col pénètrent dans le vagin. La plupart du temps les cornules ne sont que partiellement retournées. Elles reposent sur un bord poilu (HF, Fig. 2) entre la chambre de l'aiguillon et la poche copulatrice et se recourbent dorsalement vers l'aiguillon. L'aire pileuse triangulaire dorsale (TF, Fig, $2 d$ et 4) est située au-dessus du bord poilu. La grande aire pileuse ventrale (RF, Fig. 2d et 4) s'appuie contre l'appareil venimeux.

\section{Position de l'appareil venimeux}

L'appareil venimeux est déplacé dorsalement et vers le crâne par rapport à la position normale (Fig. 2). En même temps les plaques de l'appareil venimeux tournent autour de leur axe dorso-ventral. La fine enveloppe plissée de la poche copulatrice est étirée de sorte que le vagin présente une ouverture agrandie.

\section{Discussion}

On discute le schéma suivant du processus naturel de copulation :

Après avoir saisi l'abdomen de la reine dans une étreinte dorso-ventrale entre les pattes antérieures et postérieures, le mâle examine l'extrémité de son abdomen avec le champ sensoriel de sa propre région génitale (RuTTNER, 1962). Il ne dévagine d'abord que la région pileuse ventrale (RF, Fig. 2d) qui devient attachée au signe de fécondation (Fig. 3). Tandis qu'a lieu l'éversion, le signe de fécondation est tourné à l'intérieur de la reine, jusqu’à ce que les plaques chitineuses 
dorsales du bulbe (CD) se retrouvent sous l'aiguillon. Au cours de ce processus le mâle devient paralysé et bascule en arrière. Lors de la copulation avec des reines attachées, l'éversion s'arrête généralement à ce stade. Les sclérites du col ont alors pénétré dans le vagin. Le vestibule est rempli par le bulbe et ses plaques chitineuses, du mucus et du sperme (Fig. 4a). Le vestibule et le signe de fécondation $\mathrm{du}$ prédécesseur doivent distendre fortement la poche copulatrice. L'appareil venimeux est déplacé, l'aiguilion est situé entre le tergite et l'endophallus (Fig. 2).

Dans le film sur l'accouplement (KoENiger, 1981), l'éversion ne se produit que quelque temps plus tard. Puisque le mâle est déjà paralysé, une participation active de la reine est manifestement nécessaire. Dans des conditions expérimentales l'éjaculation du sperme a lieu peu de temps avant l'éversion du bulbe (Fig. 4b, Woyke et RuttNer).

Le processus d'accouplement s'arrête à nouveau lorsque le bulbe et le lobe feuilleté sont encore attachés à la reine (Fig. 5). Puis les cornules sont complètement retournées, leur substance orange reste collée dans la reine. Le signe de fécondation du prédécesseur est attaché à l'aire pileuse ventrale du vestibule. Une participation active de la reine est à nouveau nécessaire pour détacher le mâle de la reine. Après la séparation le bulbe est totalement retourné (TriASKo, 1957, et $a$ l.), son propre signe de fécondation reste attaché à la reine.

La structure complexe de l'endophallus du mâle semble ne pas être seulement une adaptation à la liaison ferme avec la reine durant la copulation en vol ; elle peut être aussi considérée comme une adaptation pour combler la distance entre l'ouverture de l'abdomen et l'orifice du vagin, sans être blessé par l'aiguillon.

\section{SUMMARY}

\section{MORPHO-FUNCTIONNAL STUDY OF THE MATING IN THE HONEYBEE (APIS MELLIFERA L.)}

Free flying drones could mate with queens, whose stingchambers were held open by blocking the articulation between the last tergite and sternite (KOENIGER et al., 1979). In this experiment the mating process in most cases stopped after the eversion of the endophallus up to the sclerites of the cervix (SC, Fig. 2d and 4a). In this position, the pair was connected so strongly that it could be frozen in copula $\left(-80^{\circ} \mathrm{C}\right)$. For preparation, the pair was embedded in Agar jelly to avoid a displacement of the endophallus.

\section{Results}

1. Position of the endophallus

The sclerites of the cervix penetrate into the vagina. The cornua are usually only partly everted. They lie on a hairy rim (HF, fig. 2) between the sting chamber and the bursa copulatrix, and bend dorsally towards the sting. The hairy dorsal triangular field (TF, fig. $2 \mathrm{~d}$ and 4) lays above this rim. The bigger hairy ventral field (RF, fig. $2 d$ and 4 ) presses against the sting apparatus.

\section{Position of the sting apparatus}

The sting apparatus is displaced dorsally and cranially compared to the normal position (Fig. 2). At the same time the plates of the sting apparatus are turned around their dorso-ventral axes. The skinny wrinkled cover of the bursa copulatrix is extended in a way such that the vagina shows an enlarged opening.

\section{Discussion}

The following scheme of the natural copulation process is discussed :

After grasping the queen's abdomen in a dorso-ventral clasp between the fore and hind legs, the drone examines the tip of her abdomen with his genital sense field (Rutrner, 1962). First he 
everts only the ventral hairy field (RF, Fig. 2d) which becomes attached to the mating sign (Fig. 3). While eversion proceeds, the mating sign is turned within the queen, until the dorsal chitinized plates of the bulbus (CD) lie under the sting. During this process, the drone becomes paralyzed and swings backwards. In this position the eversion usually stops during mating with fixed queens. Then the sclerites of the cervix (SC. Fig. 2d) have penetrated into the vagina. The vestibulum is filled with the bulbus and its chitinized plates, with mucus and sperm (fig. 4a). The vestibulum together with the mating sign of the predecessor must extend the bursa copulatrix quite strongly. The sting apparatus is displaced, the sting is situated then between the tergite and the endophallus (Fig. 2).

In the film on mating (Koeniger, 1981), the eversion only proceeds after some time. Since the drone is already paralyzed, some active part of the queen is obviously necessary. The ejaculation of sperm occurs under experimental conditions shortly before the eversion of the bulbus (Fig. $4 \mathrm{~b}$, WOYKE and RUTTNER, 1958).

The mating process stops again when bulbus and fimbriate lobe are still fixed in the queen (Fig. 5). Then the cornua are fully everted, and their sticky orange layer is left in the queen. The mating sign of the predecessor is tangled to the ventral hairy field of the vestibulum. Again some active part of the queen seems necessary to detach the drone from the queen. After separation, the bulbus is everted totally (TriasKo, 1957, u.a.) the drone's own mating sign is left in the queen.

The complicated structure of the endophallus of the drone seems to be not only an adaption to a strong connection with the queen during the copulation in free flight, but also can be regarded as an adaption to bridge the distance between the opening of the abdomen and the orifice of the vagina without being injured by the sting.

\section{LITERATUR}

Arnhart L., 1935. - Ein von der Bienenkönigin bei der Begattung gespießter Penis. Arch. Bienenkd., 16, $126-128$.

Arnhart L., 1938. - Bau der Kloake der Königin von Apis mellifica und Bedeutung derselben für die Begattung. Arch. Bienenkd., 19, 49-69.

Buttel-Reepen H. v., 1919. - Eine seltsame Verhinderung normaler Eierablage. Wie werden die Eier befruchtet? Bienenwirtsch. Zentralbl., 55 (21/22).

Bishop G.P., 1920. - Fertilization in the honeybee. J. exp. Zool,, 31, 225-286.

Fyg W., 1947. - Von der Begattung und den Ausflügen der Bienenkönigin. Schweiz. Bienen-Ztz., 70, 76-86.

GARY N., 1962. - Chemical mating attractants in the queen honey bee. Science N.Y., 136, 773-774.

GARY N. and MARSTON J., 1971. - Mating behaviour of drone honey bees with queen models (Apis mellifera). Anim. Behar., 19, 299-304.

Koeniger G., 1981. - Entfernung des Begattungszeichens durch den sich paarenden Drohn. XXVIII Int. Beekeep. Congr. Acapulco, Mexiko, 235-237.

Koeniger G., Koeniger N. und Fabritius M., 1979. - Some detailed observations of mating in the honeybee. Bee World, 60, 53-57.

Pain J. et Ruttner F., 1963. - Les extraits des glandes mandibulaires des reines d'abeilles attirent les mâles, lors du vol nuptial. CR. Açad. Sci, Paris Ser. D, 256, 512-515.

RenNer M., und Vierling G., 1977. - Die Rolle des Taschendrüsenpheromons beim Hochzeitsflug der Bienenkönigin. Behav. Ecol. Sociobiol., 2, 329-338.

Roserts W.C., 1944. - Multiple mating of queen bees proved by progeny and flight tests. Bee Glean. Cult., 72, 281-283.

RUTTNER F., 1962. - Bau und Funktion des peripheren Nervensystems an den Fortpflanzungsorganen der Drohnen. Ann. Abeille, 5, 5-58. 
Ruttner F., 1954. - Mehrfache Begattung der Bienenkönigin. Zool. Anz., 153, 99-105.

RutTner F., 1975. - Ein metatarsaler Haftapparat bei den Drohnen der Gattung Apis (Hymenoptera : Apidae). Entomol. Germ., 2, 22-29.

Ruttner F. und Ruttner H., 1972. - Untersuchungen über die Flugaktivität und das Paarungsverhalten der Drohnen : 5. Drohnensammelplätze und Paarungsdistanz. Apidologie, 3, 203-232.

SNodgrass R.E., 1956. - Anatomy of the honeybee. Ithaca, New York Comst. Publ. Assoc.

TABER S. III, 1954. - The frequency of multiple mating of queen honey bees. J. Econ. Entomol., 47, 995-998.

Triasko V.V., 1956. - Multiple matings of queen bees. Pchelovodstvo, (1), $50-54$ (in russian).

Triasko V.V. 1957. - Über Drohnen, welche sich mit Königinnen paaren. Pchelovodstvo, 34 (12), 29-31 (in Russisch).

WoYke J., 1955. - Multiple mating of the honeybee queen (Apis mellifica L.) in one nuptial flight. Bull. Acad. Pol. Sci, II (3), 175-180.

Woyke J., and Ruttner F. 1958. - An anatomical study of the mating process in the honeybee. Bee World, 39, 3-18.

Zander E., 1922. - Der Ball der Biene. Stuttgart. 